\title{
Venous thromboembolism in patients with gout and the impact of hospital admission, disease duration and urate-lowering therapy
}

\author{
Alyshah Abdul Sultan PhD, Sara Muller PhD, Rebecca Whittle PhD, Edward Roddy PhD, Christian Mallen PhD, \\ Lorna Clarson PhD
}

Cite as: CMAJ 2019 June 3;191:E597-603. doi: 10.1503/cmaj.180717

\begin{abstract}
BACKGROUND: Systemic inflammatory diseases have been associated with increased risk of venous thromboembolism. We aimed to quantify the risk of venous thromboembolism in patients with gout, the most common inflammatory arthritis, and to assess how disease duration, hospital admission and uratelowering therapy affect this risk.
\end{abstract}

METHODS: We used data from the population-representative, Englandbased Clinical Practice Research Datalink linked to Hospital Episode Statistics, to identify incident gout cases between 1998 and 2017. We matched cases individually to 1 control without gout on age, gender, general practice and followup time. We calculated absolute and rel- ative risks of venous thromboembolism, stratified by age, gender and hospital admission. Among those with gout, we assessed the risk of venous thromboembolism by exposure to urate-lowering therapy.

RESULTS: We identified 62234 patients with incident gout matched to 62234 controls. Gout was associated with higher risk of venous thromboembolism compared with controls (absolute rate $37.3[95 \%$ confidence interval $(\mathrm{Cl}) 35.5-$ 39.3] v. 27.0 [95\% Cl 25.5-28.9] per 10000 person-years, adjusted hazard ratio $[\mathrm{HR}] 1.25,95 \% \mathrm{Cl} 1.15-1.35)$. The excess risk in patients with gout, which was sustained up to a decade after diagnosis, was present during the time out- side hospital stay (adjusted HR 1.30, 95\% Cl 1.18-1.42), but not during it (adjusted HR 1.01, 95\% Cl 0.83-1.24). The risk of venous thromboembolism was similar among patients prescribed versus not prescribed urate-lowering therapy (incidence rate ratio 1.04 , $95 \% \mathrm{Cl} 0.89-1.23)$.

INTERPRETATION: Gout was associated with higher risk of venous thromboembolism, particularly when the patient was not in hospital and regardless of exposure to urate-lowering therapy. Although the observed excess risk may not be sufficient to warrant preventive intervention, clinical vigilance may be required when caring for these patients.
V enous thromboembolism is known to be a major preventable cause of morbidity and mortality worldwide. ${ }^{1}$ In order to reduce the morbidity and mortality associated with venous thromboembolism, it is important to reduce the occurrence of the condition. ${ }^{2}$ Previous studies have highlighted rheumatological diseases, including rheumatoid arthritis and systematic lupus erythematosus, to be important predictors of venous thromboembolism, the risk of which is increased up to five-fold in these diseases. ${ }^{3,4}$ Chronic inflammatory processes are considered to increase the risk of venous thromboembolism through various mechanisms (e.g., upregulation of procoagulants, suppression of fibrinolysis). ${ }^{5,6}$

Gout is the most common inflammatory arthritis, affecting $2.4 \%$ of adults, ${ }^{7}$ and typically affects older populations with an already high baseline risk for venous thromboembolism. ${ }^{1}$ It has been suggested that serum urate (the biochemical prerequisite for the development of deposition of monosodium urate crystals and of gout) has a pro-inflammatory effect and can initiate, amplify and sustain inflammatory responses. A linear relation between serum urate and risk of venous thromboembolism has been shown. ${ }^{5}$ In addition to the effect of hyperuricemia, a potent inflammatory reaction is triggered by the deposition of monosodium urate crystals in joints and soft tissues, which is pathognonomic of gout. Historically, gout has been viewed as an episodic inflammatory condition, but it is now considered to be a chronic inflammatory arthritis in which inflammation persists between clinical flares.

Few studies have assessed how these effects of hyperuricemia, deposition of monosodium urate crystals and inflammation translate into risk of venous thromboembolism in patients with gout. The only population-based study ${ }^{8}$ on the subject reported a $66 \%$ increased risk of deep vein thrombosis in patients with 
clinically diagnosed gout, but failed to take into account episodes of hospital admission - one of the biggest risk factors for venous thromboembolism ${ }^{9}$ - along with other known risk factors (e.g., body mass index, smoking status), or to assess the potential impact of urate-lowering therapy.

The aim of our study was to assess the overall occurrence of venous thromboembolism in patients with gout in a populationbased sample and to separate out the effects of hospital stay and exposure to urate-lowering therapy on the risk of venous thromboembolism.

\section{Methods}

\section{Data source and study population}

We used the Clinical Practice Research Datalink (CPRD), a large database containing UK primary care medical records of anonymized patients. The CPRD covers $7 \%$ of the UK population and is representative of the general UK population in terms of age and gender distribution. The Hospital Episodes Statistics (HES) data set contains details of all National Health Service (NHS) inpatient care, outpatient appointments and emergency attendance in England. The CPRD and HES databases have been linked, and for each patient, both primary and secondary care data are therefore available. The linkage is performed by a trusted third party using NHS number, date of birth and gender. The CPRD-HES linked data cover 3\% of the total English population and are representative of the general UK population. ${ }^{10}$

We identified individuals in the CPRD with first-ever recorded diagnosis of gout between 1998 and 2016, using previously published methods. ${ }^{7}$ Briefly, gout diagnosis was based on a medical code assigned by the general practitioner, which has been previously validated in CPRD and has a positive predictive value of $90 \% .{ }^{11}$ We assigned each patient an index date corresponding to the date of their gout diagnosis and randomly matched them to 1 control, without gout diagnosis or urate-lowering therapy, on age $( \pm 5 \mathrm{yr}$ ), gender, follow-up time available in CPRD $( \pm 3 \mathrm{yr}$ ) and general practice. Follow-up commenced from the index date. We excluded those with a history of venous thromboembolism, or less than 1 year of follow-up after the index date.

\section{Study outcome}

We based the diagnosis of venous thromboembolism on medical codes assigned by a general practitioner. We also identified cases of venous thromboembolism recorded in secondary care and in the Office of National Statistics death register. We considered a diagnosis of venous thromboembolism to be valid only if it was accompanied by anticoagulant prescription within 90 days of the event, or if death was recorded within 30 days of the diagnosis. We considered venous thromboembolism recorded as the underlying cause of death in the Office of National Statistics death register to be valid without any further confirmation. This algorithm has been previously validated in CPRD with an accuracy (positive predictive value) of $84 \% .{ }^{12}$ For the purpose of this study, we excluded women with pregnancy-related venous thromboembolism events based on medical codes.

\section{Hospital admission}

To assess the effect of hospital admission (for any reason) on the risk of venous thromboembolism, we obtained information on all hospital admissions that were not for venous thromboembolism and that lasted 1 or more days. The overall person-time in the study was broadly divided into "in-hospital period" (time between admission and discharge) and "ambulatory period" (time not associated with hospital stay, including entire persontime of patients never admitted to hospital during the study period). Appendix 1 (available at www.cmaj.ca/lookup/suppl/ doi:10.1503/cmaj.180717/-/DC1) shows the composition of person-time. The ambulatory period was further divided into "before hospital admission" (time between the index date and first hospital admission) and "postdischarge" period.

To assess the risk of venous thromboembolism arising from hospital stay, we needed to ensure inpatient venous thromboembolism was the consequence rather than the cause of hospital admission. Therefore, we manually reviewed initial hospital diagnoses and a 7-day general practice medical history of all venous thromboembolism events that occurred during the in-hospital period, as previously described..$^{13,14} \mathrm{~A}$ venous thromboembolism event was deemed to be hospital-related only if there was no evidence of venous thromboembolism or related symptom (e.g., chest pain for pulmonary embolism) in the initial hospital diagnoses of the index hospital admission or in the days preceding admission.

\section{Exposure to urate-lowering therapy}

Among those with gout, we extracted information on first exposure to urate-lowering therapy after gout diagnosis. We calculated the duration of urate-lowering therapy based on quantity prescribed and numeric daily dose. We considered patients to be "exposed to urate-lowering therapy" only if they were on uratelowering therapy for more than 6 months from the date of prescription. We considered patients not on urate-lowering therapy or prescribed less than 6 months of urate-lowering therapy to be "not exposed to urate-lowering therapy" (Figure 1). This was based on previous literature ${ }^{15}$ and expert consensus, as the usual practice for prescribing allopurinol is to start the medication at a low dose and increase the dose gradually, as it can take several months to escalate the dose to lower serum urate sufficiently to achieve the biochemical target level. Each patient exposed to urate-lowering therapy was individually matched to 1 patient not exposed to gout, on age, gender and year of prescription. For analysis of urate-lowering therapy, follow-up started from the date of first prescription of urate-lowering therapy (randomly assigned date for unexposed patients within 1-yr accrual blocks).

\section{Other potential confounding factors}

For each individual, we extracted information on body mass index (BMI), alcohol consumption, smoking status and Charlson comorbidity index, using the most recent recording before the study end date. We defined information on socioeconomic status based on the location of the general practice at which the patient was registered (quintiles by rank of Indices of Multiple Deprivation ${ }^{16}$ ). We also extracted information on diabetes and hypertension and previous prescriptions for acetylsalicylic acid (ASA) or thiazide. 


\section{Statistical analysis}

We calculated absolute rates of venous thromboembolism per 10000 person-years and 95\% confidence intervals $(\mathrm{Cl})$ for patients in the gout and control groups. We stratified these by age, gender and calendar year. We calculated hazard ratios (HR) using a Cox proportional hazards regression model, adjusting for the stated confounding factors. We categorized those with missing BMI status as a separate category and included them in the analysis, as we assumed BMI to be not missing at random. We tested the proportional hazards assumption using Schoenfeld residuals. To assess the impact of hospital admission, we calculated the absolute and relative rate of venous thromboembolism during in-hospital and ambulatory periods. To assess the impact of disease duration, we calculated the risk of venous thromboembolism in the years after gout diagnosis and compared this risk to that in controls using incidence rate ratios (IRRs) calculated using a Poisson regression analysis. Similarly, among those with gout, we assessed the risk of venous thromboembolism by exposure to urate-lowering therapy. Based on the sample of 62234 patients with gout matched to the same number of control patients and assuming the annual incidence of venous thromboembolism to be $0.2 \%$, we had more than $80 \%$ power to detect a hazard ratio of 1.38 at $5 \%$ level of significance.

We performed all statistical analyses using Stata version 14.

\section{Ethics approval}

This study was approved by the CPRD in-house Independent Scientific Advisory Committee reference number: reference number: 15_214RA.

\section{Results}

We identified 62234 patients with incident gout who were individually matched to the same number of controls from the general population (Figure 1). Compared with control patients, patients with gout had higher BMIs and more comorbidities overall, and were more likely to have hypertension, use ASA and thiazides, and consume more units of alcohol per week, but were less likely to be current smokers (Table 1).

\section{Risk of venous thromboembolism}

Compared with control patients, those with incident gout had a higher absolute rate of venous thromboembolism $(37.3[95 \% \mathrm{Cl}$ 35.5-39.3] v. 27.0 [95\% Cl 25.5-28.9] per 10000 person-years) and an excess risk after adjusting for baseline covariates (adjusted hazard ratio [HR] $1.2595 \% \mathrm{Cl} 1.15-1.35$ ) (Table 2). This finding was consistent for both men (adjusted $\mathrm{HR} 1.20,95 \% \mathrm{Cl}$ 1.09-1.33) and women (adjusted HR 1.32, 95\% Cl 1.14-1.52).

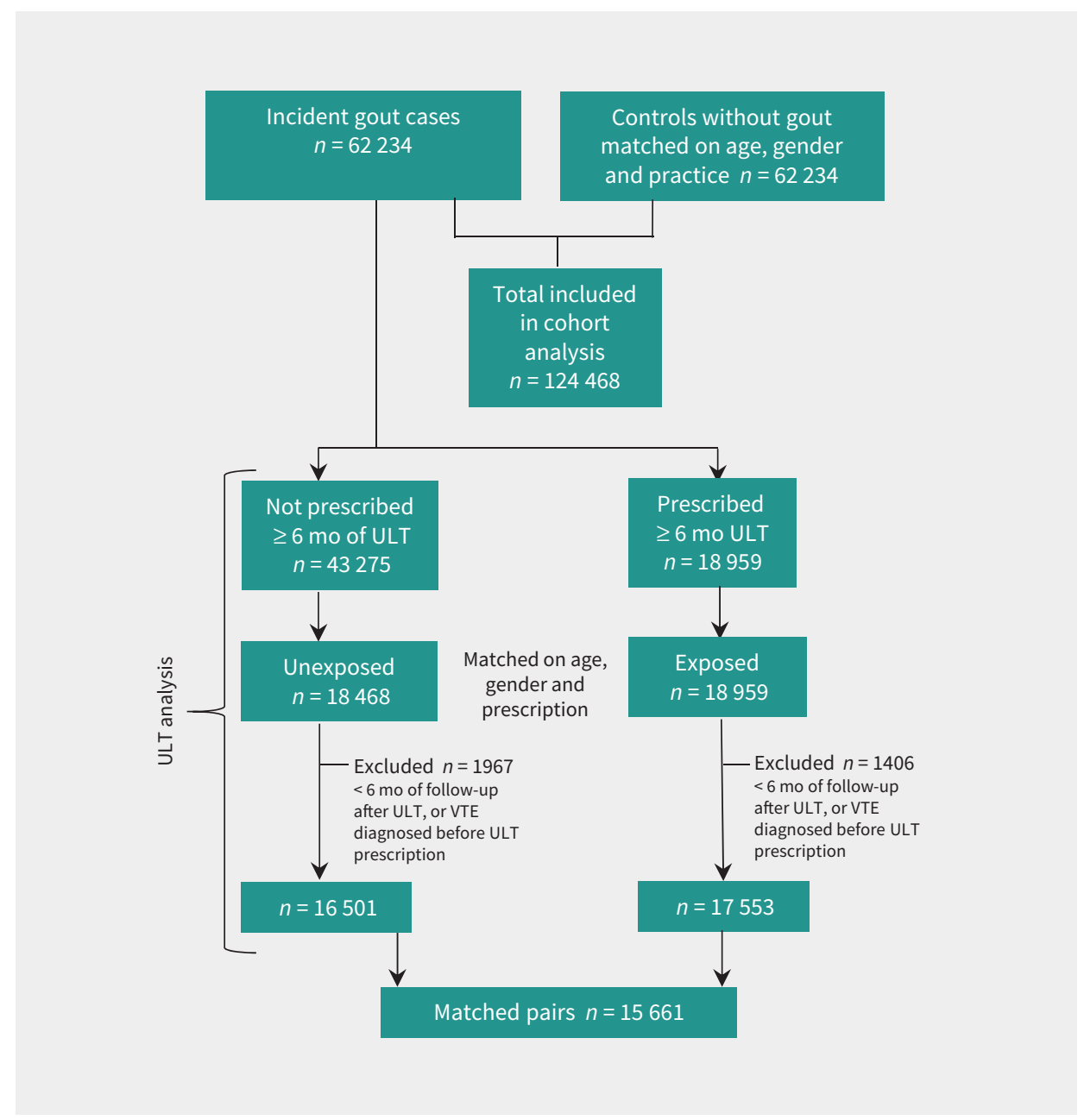

Figure 1: Flow diagram of data structure. Note: ULT = urate-lowering therapy, VTE = venous thromboembolism. 
Although the absolute rate of venous thromboembolism increased with age ( $p$ value for trends $>0.001$ ), the excess risk was particularly high for younger patients (aged $<50 \mathrm{yr}$, adjusted HR 1.79, 95\% Cl 1.30-2.48).

\section{Impact of admission to hospital}

In total, 142474 unique hospital admissions occurred after the index date. Initially, 529 cases of venous thromboembolism occurred during in-hospital stay and were deemed to be the consequence of a hospital stay. However, after reviewing medical records for those patients, we recategorized 121 of those cases of venous thromboembolism as the cause of hospital admission. We found no difference in the rate of venous thromboembolism between patients with gout and control patients during the in-hospital period (IRR $=1.01,95 \% \mathrm{Cl} 0.83-$ 1.24; Table 3). In contrast, the rate of venous thromboembolism was higher for patients with gout during the ambulatory period $(\mathrm{IRR}=1.30,95 \% \mathrm{Cl} 1.18-1.42)$ compared with the control group.

Table 1: Basic characteristics of the study population

\section{Characteristic}

Age at index, mean \pm SD

Male

Median follow-up (IQR)

Body mass index

Normal (18.5-24.9)

Underweight (<18.5)

Overweight (25.29.9)

Obese (>30)

Missing

Smoking status

Never or ex-smoker

Current smokers

Charlson index (IQR)

0

$1-2$

$3-4$

$>5$

\section{Deprivation}

1 (least deprived)

2

3

4

5 (most deprived)

Missing

Diabetes

Hypertension

Acetylsalicylic acid use

Thiazide use

Alcohol consumption

Never or ex-drinker

Current ( $<10$ units/wk)

Current ( $\geq 10$ units/wk)

Missing

\section{No. of control patients (\%) *}

$$
n=62234
$$

$62.3 \pm 15.1$

45951 (73.8)

$5.7(3.1-9.1)$

$19602(31.5)$

1393 (2.2)

22149 (35.6)

12333 (19.8)

6757 (10.9)

53234 (85.5)

$9000(14.5)$

30429 (48.9)

$18113(29.1)$

8312 (13.4)

$5380(8.6)$

15438 (24.8)

15537 (25.0)

12639 (20.3)

$10770(17.3)$

7788 (12.5)

$62(0.1)$

6004 (9.6)

$13172(21.2)$

13235 (21.3)

11936 (19.2)

8530 (13.7)

28198 (45.3)

$14096(22.6)$

$11410(18.3)$
No. of patients with gout (\%)*

$n=62234$

$62.4 \pm 15.1$

45951 (73.8)

$5.7(3.1-9.1)$

12312 (19.8)

694 (1.1)

23340 (37.5)

22285 (35.8)

3603 (5.8)

55764 (89.6)

0.123

6470 (10.4)

$23633(38.0)$

0.272

$18297(29.4)$

11088 (17.8)

9216 (14.8)

15211 (24.4)

0.009

$15552(25.0)$

12739 (20.5)

10880 (17.5)

7794 (12.5)

$58(0.1)$

7677 (12.3)

0.086

$21318(34.3)$

0.297

$18505(29.7)$

0.195

$21033(33.8)$

0.335

7729 (12.4)

0.269

20898 (33.6)

7602 (12.2)

Note: IQR = interquartile range, $\mathrm{SD}=$ standard deviation

*Unless stated otherwise.

†Standardized difference $=$ difference in means or proportion divided by standard error; imbalance defined as absolute value $>0.10$ (small effect size) . 
Impact of disease duration

In terms of the risk of venous thromboembolism in relation to time since gout diagnosis, we observed higher rates of venous thromboembolism compared with controls within 1 year of gout diagnosis (34 v. 19 per 10000 person-years), which remained consistently high up to 10 years after diagnosis (Table 3). However, the excess risk was not statistically different between patients with gout and control patients beyond 10 years since gout diagnosis.

Table 2: Absolute rate of venous thromboembolism per 10000 person-years and hazard ratios

\begin{tabular}{|c|c|c|c|c|c|c|c|c|}
\hline \multirow[b]{2}{*}{ Variable } & \multirow[b]{2}{*}{$N$} & \multicolumn{2}{|c|}{ Control patients } & \multicolumn{3}{|c|}{ Patients with gout } & \multirow[b]{2}{*}{$\begin{array}{l}\text { Unadjusted } \\
\text { HR }(95 \% \mathrm{CI})\end{array}$} & \multirow[b]{2}{*}{$\begin{array}{c}\text { Adjusted HR* } \\
(95 \% \mathrm{CI})\end{array}$} \\
\hline & & $\begin{array}{l}\text { Person- } \\
\text { years }\end{array}$ & Rate $(95 \% \mathrm{Cl})$ & $N$ & $\begin{array}{l}\text { Person- } \\
\text { years }\end{array}$ & $\begin{array}{l}\text { Rate } \\
(95 \% \mathrm{Cl})\end{array}$ & & \\
\hline Overall & 1071 & 396095 & $27.0(25.5-28.9)$ & 1481 & 396990 & $37.3(35.5-39.3)$ & $1.38(1.28-1.49)$ & $1.25(1.15-1.35)$ \\
\hline \multicolumn{9}{|l|}{ Gender } \\
\hline Male & 721 & 297075 & $24.3(22.6-26.1)$ & 953 & 298577 & $31.9(30.0-34.0)$ & $1.31(1.19-1.45)$ & $1.20(1.09-1.33)$ \\
\hline Female & 350 & 99020 & $35.3(31.8-39.3)$ & 528 & 98413 & $53.7(49.3-58.4)$ & $1.52(1.33-1.74)$ & $1.32(1.14-1.52)$ \\
\hline \multicolumn{9}{|l|}{ Age, yr } \\
\hline$<50$ & 63 & 97437 & $6.5(5.1-8.3)$ & 126 & 97224 & $13.0(10.9-15.4)$ & $2.00(1.48-2.71)$ & $1.79(1.30-2.48)$ \\
\hline $50-59$ & 140 & 86944 & $16.1(13.6-19.0)$ & 214 & 86633 & $24.7(21.6-28.2)$ & $1.53(1.24-1.90)$ & $1.40(1.12-1.75)$ \\
\hline $60-69$ & 269 & 93635 & $28.7(25.5-32.4)$ & 393 & 93734 & $41.9(38.0-46.3)$ & $1.46(1.25-1.70)$ & $1.25(1.06-1.47)$ \\
\hline $70-79$ & 377 & 81384 & $46.3(41.9-51.2)$ & 482 & 82440 & $58.5(53.5-63.9)$ & $1.26(1.10-1.44)$ & $1.18(1.02-1.36)$ \\
\hline$>80$ & 222 & 36694 & $60.5(53.0-69.0)$ & 266 & 36960 & $72.0(63.8-81.2)$ & $1.19(0.99-1.42)$ & $1.16(0.96-1.39)$ \\
\hline \multicolumn{9}{|c|}{ Deprivation index quintiles } \\
\hline 1 (least deprived) & 251 & 101402 & $24.8(21.9-28.0)$ & 345 & 99888 & $34.5(31.0-38.4)$ & $1.40(1.19-1.64)$ & $1.31(1.10-1.54)$ \\
\hline 2 & 271 & 99615 & $27.2(24.2-30.6)$ & 360 & 99706 & $36.1(32.6-40.0)$ & $1.33(1.13-1.55)$ & $1.22(1.03-1.44)$ \\
\hline 3 & 213 & 79824 & $26.7(23.3-30.5)$ & 308 & 80398 & $38.3(34.3-42.8)$ & $1.44(1.21-1.71)$ & $1.28(1.06-1.53)$ \\
\hline 4 & 192 & 66674 & $28.8(25.0-33.2)$ & 283 & 67770 & $41.8(37.2-47.0)$ & $1.45(1.20-1.74)$ & $1.29(1.07-1.57)$ \\
\hline 5 (most deprived) & 143 & 48219 & $30.0(25.2-35.0)$ & 183 & 48879 & $37.4(32.4-43.3)$ & $1.26(1.01-1.57)$ & $1.09(0.87-1.37)$ \\
\hline
\end{tabular}

Note: $\mathrm{Cl}$ = confidence interval, $\mathrm{HR}=$ hazard ratio.

*Adjusted for age, gender, Charlson index calendar year, smoking status, hospital admission, deprivation, hypertension, diabetes, acetylsalicylic acid use, thiazide use, alcohol consumption and body mass index.

Table 3: Absolute and relative rate of venous thromboembolism by hospital admission and time after gout diagnosis

\begin{tabular}{|c|c|c|c|c|c|c|c|c|}
\hline \multirow[b]{2}{*}{ Variable } & \multicolumn{3}{|c|}{ Control patients } & \multicolumn{3}{|c|}{ Patients with gout } & \multirow[b]{2}{*}{$\begin{array}{c}\text { IRR } \\
(95 \% \mathrm{CI})\end{array}$} & \multirow{2}{*}{$\begin{array}{c}\text { IRR } \\
\text { (95\% CI) } \\
\text { adjusted* }\end{array}$} \\
\hline & $N$ & $\begin{array}{c}\text { Person- } \\
\text { years }\end{array}$ & Rate $(95 \% \mathrm{CI})$ & $N$ & $\begin{array}{c}\text { Person- } \\
\text { years }\end{array}$ & Rate $(95 \% \mathrm{CI})$ & & \\
\hline \multicolumn{9}{|l|}{ Hospital admission } \\
\hline In-hospital period & 178 & 2145 & $829.8(716.3-961.1)$ & 230 & 2787 & $825.2(725.1-939.0)$ & $0.99(0.82-1.21)$ & $1.01(0.83-1.24)$ \\
\hline Ambulatory period & 893 & 393950 & $22.7(21.2-24.2)$ & 1251 & 394203 & $31.7(30.0-33.5)$ & $1.40(1.28-1.53)$ & $1.30(1.18-1.42)$ \\
\hline Before hospital admission & 439 & 306627 & $14.3(13.0-15.7)$ & 574 & 283368 & $20.3(18.7-22.0)$ & $1.41(1.25-1.60)$ & $1.37(1.19-1.56)$ \\
\hline Postdischarge period & 454 & 87323 & $52.0(47.4-57.0)$ & 677 & 110835 & $61.1(56.5-65.9)$ & $1.17(1.04-1.32)$ & 1.15 \\
\hline \multicolumn{9}{|c|}{ Time after gout diagnosis in years } \\
\hline 1 & 119 & 62179 & $19.1(16.0-22.9)$ & 208 & 62134 & $33.5(29.2-38.4)$ & $1.75(1.40-2.19)$ & $1.70(1.35-2.16)$ \\
\hline$>1-6$ & 611 & 221787 & $27.5(25.5-29.8)$ & 817 & 222456 & $36.7(34.3-39.3)$ & $1.33(1.20-1.48)$ & $1.22(1.10-1.37)$ \\
\hline$>6-10$ & 231 & 79517 & $29.1(25.5-33.1)$ & 320 & 79826 & $46.1(35.9-44.7)$ & $1.38(1.17-1.63)$ & $1.28(1.06-1.52)$ \\
\hline$>10$ & 110 & 32613 & $33.7(28.0-40.7)$ & 136 & 32574 & $41.7(35.3-49.4)$ & $1.24(0.96-1.59)$ & $1.06(0.81-1.38)$ \\
\hline
\end{tabular}


Table 4: Absolute risk of venous thromboembolism per 10000 by exposure to urate-lowering therapy

$\begin{aligned} & \text { Variable } \\ & \text { All periods }\end{aligned}$
$\begin{aligned} & \text { Not exposed to ULT } \\ & \text { Exposed to ULT }\end{aligned}$
$\begin{aligned} & \text { Nears } \\ & \text { IRR (95\% CI) } \\ & \text { adjusted }\end{aligned}$

\section{Impact of urate-lowering therapy}

Of the total patients with gout, 30\% received at least 6 months of urate-lowering therapy during the follow-up period. After applying exclusion criteria (Figure 1), we included 15661 matched pairs in the analysis. Patients receiving at least 6 months of urate-lowering therapy had higher BMIs and more comorbidities overall and were more likely to have hypertension or diabetes and to use ASA (Appendix 2, available at www.cmaj.ca/lookup/suppl/doi:10.1503/cmaj.180717/-/ DC1). However, after adjustment for baseline characteristics, such patients showed no difference in the risk of venous thromboembolism overall (adjusted IRR 1.04, 95\% Cl 0.89-1.23) (Table 4) or in the in-hospital and ambulatory periods (data not shown).

\section{Interpretation}

Using data from a large, nationally representative cohort, we quantified the risk of venous thromboembolism among patients with gout. Compared with the those in the control group, patients with gout were $25 \%$ more likely to develop venous thromboembolism after their diagnosis. Although the risk of venous thromboembolism increased with age, the excess risk was higher for younger patients (aged $<50 \mathrm{yr}$ ). The excess risk of venous thromboembolism remained consistently high up to a decade after diagnosis and was particularly observed during the time not associated with hospital admission. Finally, among patients with gout, we found no difference in the risk of venous thromboembolism by prescription of urate-lowering therapy. Our findings remained unchanged when we stratified our analysis by hospital admission.

A major strength of this study is that we have used large, routinely collected data to conduct our analysis. This allowed us to assess the risk of venous thromboembolism in patients with gout with minimum information bias, as identification of outcome was independent of gout status. Using large, representative data means that our findings are generalizable to not only the UK population, but also to others with similar health care systems. Further strengths include the large number of venous thromboembolism events and long duration of follow-up.

Few studies have reported the risk of venous thromboembolism in patients with gout. ${ }^{5,8,17} \mathrm{~A}$ recent hospital-based case-control study ${ }^{17}$ from Japan reported a ninefold increased risk of pulmonary embolism in patients with gout or hyperuricemia, but the number of events was very small and the study was therefore underpowered to provide precise estimates ( $\mathrm{Cl}$ between 1.6 and 46.0). In contrast, another study from the United States ${ }^{5}$ reported no statistically significant association between gout and subsequent venous thromboembolism. The positive association between gout and venous thromboembolism in the previous study may have been masked, as gout ascertainment was conditional on survival and participation in follow-up visits occurring more than 5 years after initial recruitment, which may have resulted in selection bias. Although the relative risk in our study is slightly lower, it may be explained by the difference in the study population and more comprehensive consideration of confounding factors that include $\mathrm{BMI}$, smoking status and hospital admission in our study. Our finding of higher relative risk observed in the younger population has been previously reported. ${ }^{8}$ Allopurinol has been shown to decrease cardiovascular risk, ${ }^{18}$ but we found no statistically significant association between prescription of urate-lowering therapy and venous thromboembolism. This finding may be owing to the fact that in the UK, allopurinol is often prescribed at a low dose by primary care practitioners and most patients do not reach target serum uric acid levels. ${ }^{19}$

The excess risk of venous thromboembolism associated with gout in this study is small compared with risks reported in other inflammatory rheumatological diseases, such as rheumatoid arthritis. ${ }^{6}$ This may reflect the fluctuant nature of inflammation in gout with intense acute inflammation during flares and lower-grade inflammation in the intercritical period between flares, or a differing cause from autoimmune disorders. Because recurrent flares are commonly unrecorded in CPRD, as patients may self-manage without consultation, we were unable to explore this concept further.

\section{Limitations}

A potential weakness of this study is the use of anonymized patient records. As we had no access to individual patients, we relied on physicians to have accurately recorded information on gout and venous thromboembolism. However, gout diagnosis has been previously validated in CPRD with high accuracy, ${ }^{11}$ and therefore it is unlikely that there is any major error in our findings owing to misclassification of our case patients. These findings are in line with another study in which $83 \%$ of cases of gout diagnosed by general practitioners were independently validated by a rheumatologist on clinical grounds. ${ }^{20}$ Similarly, our algorithm to define venous thromboembolism had also been previously validated in CPRD with a positive predictive value of $84 \%$. However, the absence of diagnosis is not validated in these data; therefore, in practice, if venous thromboembolism is more completely ascertained in patients with than those without gout, it would lead to apparent excess risk of venous thromboembolism in this group. 
Second, the results of our analysis of urate-lowering therapy may be generalizble only to those prescribed $\leq 300 \mathrm{mg}$ of allopurinol, a dose level widely used in primary care. ${ }^{19}$ We were unable to explore whether patients adhered to urate-lowering therapy, or whether target serum uric acid levels were reached in patients prescribed urate-lowering therapy, and our finding of no association may reflect suboptimal urate-lowering rather than the true effect of urate-lowering therapy. It is possible that higher doses may have significant impact on venous thromboembolism risk, for which further studies may be needed. Third, we were unable to measure adherence in our data set. However, a previous study using a similar database $(\mathrm{CPRD})^{21}$ used the proportion of days covered as a proxy to measure nonadherence. This was calculated as the number of days of prescribed medication divided by the total duration of follow-up. Finally, in the UK, all patients undergo a risk assessment to identify their risk of venous thromboembolism and bleeding on admission to hospital. This may explain our null findings during the in-hospital period and higher venous thromboembolism risk during the ambulatory period. Unfortunately, we were not able to account for thromboprophylaxis owing to the lack of information on prescriptions originating in secondary care.

\section{Conclusion}

In our large population-based study, we found that gout is associated with increased risk of first venous thromboembolism. The increased risk is independent of hospital admission and is particularly high in the younger population. Furthermore, among patients with gout, we found no difference in the risk of venous thromboembolism by prescription of urate-lowering therapy. Although our observed excess risk may not be sufficient to warrant preventive intervention on its own, there may be need for clinical vigilance in younger patients with a new diagnosis of gout, with further research needed to establish the impact of gout severity on risk of venous thromboembolism.

\section{References}

1. White RH. The epidemiology of venous thromboembolism. Circulation 2003;107(Suppl 1):14-8.

2. Heit JA, O'Fallon WM, Petterson TM, et al. Relative impact of risk factors for deep vein thrombosis and pulmonary embolism: a population-based study. Arch Intern Med 2002;162:1245-8.
3. Lee JJ, Pope JE. A meta-analysis of the risk of venous thromboembolism in inflammatory rheumatic diseases. Arthritis Res Ther 2014;16:435.

4. Yusuf HR, Hooper WC, Grosse SD, et al. Risk of venous thromboembolism occurrence among adults with selected autoimmune diseases: a study among a US cohort of commercial insurance enrollees. Thromb Res 2015;135:50-7.

5. Kubota Y, McAdams-DeMarco M, Folsom AR. Serum uric acid, gout, and venous thromboembolism: the Atherosclerosis Risk in Communities study. Thromb Res 2016;144:144-8.

6. Holmqvist ME, Neovius M, Eriksson J, et al. Risk of venous thromboembolism in patients with rheumatoid arthritis and association with disease duration and hospitalization. JAMA 2012;308:1350-6.

7. Kuo CF, Grainge MJ, Mallen C, et al. Rising burden of gout in the UK but continuing suboptimal management: a nationwide population study. Ann Rheum Dis 2015;74:661-7.

8. Huang CC, Huang PH, Chen JH, et al. An independent risk of gout on the development of deep vein thrombosis and pulmonary embolism: a nationwide, population-based cohort study. Medicine (Baltimore) 2015;94:e2140.

9. Heit JA, Melton LJ, Lohse CM, et al. Incidence of venous thromboembolism in hospitalized patients vs community residents. Mayo Clin Proc 2001;76:1102-10.

10. Herrett E, Gallagher AM, Bhaskaran K, et al. Data Resource Profile: Clinical Practice Research Datalink (CPRD). Int J Epidemiol 2015;44:827-36.

11. Meier $\mathrm{CR}$, Jick H. Omeprazole, other antiulcer drugs and newly diagnosed gout. Br J Clin Pharmacol 1997;44:175-8.

12. Lawrenson R, Todd JC, Leydon GM, et al. Validation of the diagnosis of venous thromboembolism in general practice database studies. Br J Clin Pharmacol 2000;49:591-6.

13. Grainge MJ, West J, Card TR. Venous thromboembolism during active disease and remission in inflammatory bowel disease: a cohort study. Lancet 2010;375:657-63.

14. Abdul Sultan A, West J, Tata LJ, et al. Risk of first venous thromboembolism in pregnant women in hospital: population-based cohort study from England. BMJ 2013;347:f6099.

15. Kuo CF, Grainge MJ, Mallen C, et al. Effect of allopurinol on all-cause mortality in adults with incident gout: propensity score-matched landmark analysis. Rheumatology (Oxford) 2015;54:2145-50.

16. Jordan H, Roderick P, Martin D. The Index of Multiple Deprivation 2000 and accessibility effects on health. J Epidemiol Community Health 2004;58:250-7.

17. Yamada N, Ota S, Liu Y, et al. Risk factors for nonfatal pulmonary embolism in a Japanese population: a hospital-based case-control study. Angiology 2010;61: 269-74.

18. Larsen KS, Pottegard A, Lindegaard HM, et al. Effect of allopurinol on cardiovascular outcomes in hyperuricemic patients: a cohort study. Am J Med 2016;129: 299-306.e2.

19. Cottrell E, Crabtree V, Edwards JJ, et al. Improvement in the management of gout is vital and overdue: an audit from a UK primary care medical practice. BMC Fam Pract 2013;14:170.

20. Roddy E, Zhang W, Doherty M. Concordance of the management of chronic gout in a UK primary-care population with the EULAR gout recommendations. Ann Rheum Dis 2007;66:1311-5.

21. Scheepers LEJM, Burden AM, Arts ICW, et al. Medication adherence among gout patients initiated allopurinol: a retrospective cohort study in the Clinical Practice Research Datalink (CPRD). Rheumatology (Oxford) 2018;57:1641-50.

\section{Competing interests: None declared.}

This article has been peer reviewed.

Affiliations: Arthritis Research UK Primary Care Centre (Abdul Sultan, Muller, Whittle, Roddy, Mallen, Clarson), Research Institute for Primary Care \& Health Sciences, Keele University, UK; Haywood Academic Rheumatology Centre (Roddy), Midlands Partnership NHS Foundation Trust, Stoke-on-Trent, UK

Contributors: Lorna Clarson, Edward Roddy and Christian Mallen were responsible for study concept and design; Sara Muller, Rebecca Whittle and Alyshah Abdul Sultan acquired the data; and Alyshah Abdul Sultan analyzed and interpreted the data. Alyshah
Abdul Sultan performed the statistical analysis. All authors drafted the manuscript, revised it critically for important intellectual content, gave final approval of the version to be published and agreed to be accountable for all aspects of the work.

Funding: This study was funded by the National Institute for Health Research School for Primary Care Research (NIHR SPCR, grant number 259). Christian Mallen is funded by the NIHR Collaborations for Leadership in Applied Health Research and Care West Midlands, the NIHR SPCR and a NIHR Research Professorship in General Practice, which also supports Alyshah Abdul Sultan and Rebecca Whittle (NIHRRP-2014-04-026). The views expressed are those of the authors and not necessarily those of the National Health Service, the NIHR or the Department of Health. The funder was not involved in the study design; in the collection, analysis, and interpretation of data; in the writing of the report; and in the decision to submit the article for publication.

Data sharing: Clinical Practice Research Datalink (CPRD) data were provided under a licence that does not permit data sharing. However, data can be obtained directly from CPRD under its standard conditions.

Accepted: May 2, 2019

Correspondence to: Alyshah Abdul Sultan, alyshah.sultan@hotmail.com 